\title{
Plasma Waves and Sounder (PWS) experiment onboard the Planet-B Mars orbiter
}

\author{
Takayuki Ono ${ }^{1}$, Hiroshi Oya ${ }^{1}$, Akira Morioka ${ }^{1}$, Atsushi Kumamoto ${ }^{1}$, \\ Kaori Kobayashi ${ }^{2}$, Takahiro Obara ${ }^{3}$, and Tomoko Nakagawa ${ }^{4}$ \\ ${ }^{1}$ Department of Astronomy and Geophysics, Tohoku University, Sendai 980-8578, Japan \\ ${ }^{2}$ Toyama Prefectural University, Kosugi, Toyama 939-03, Japan \\ ${ }^{3}$ Communications Research Laboratory, Hitachinaka-shi, Ibaraki 311-12, Japan \\ ${ }^{4}$ Tohoku Institute of Technology, Sendai 982, Japan
}

(Received September 24, 1997; Revised December 5, 1997; Accepted December 10, 1997)

\begin{abstract}
For the purpose of observing the Martian ionosphere under the condition of direct interaction with the solar wind by using a RF sounder together with a plasma density probe and high frequency plasma wave detectors, the Plasma Waves and Sounder (PWS) system onboard the Planet-B Mars orbiter has been developed through a proto-model (PM) which are followed by achievement of a flight-model (FM) for the Planet-B mission. The operation of the achieved system has been tested for function and stability in a simulated space environment. The PWS system is equipped with two sets of long deployable dipole antennas with a tip-to-tip length of $52 \mathrm{~m}$. The high power (600 Watts) transmitter of the sounder experiment makes it possible to measure the electron density profiles of the Martian topside ionosphere in an altitude range from 300 to $3000 \mathrm{~km}$ in a density range from $10^{2}$ to $10^{6} / \mathrm{cc}$ with a time resolution of $25 \mu \mathrm{sec}$ corresponding to a range resolution of $3.75 \mathrm{~km}$. Observations of natural plasma waves and planetary radio waves in the frequency range from $20 \mathrm{kHz}$ to $5 \mathrm{MHz}$ are planned in the passive mode operation of the PWS system, identifying all the polarization characteristics together; observations of natural plasma waves caused by the direct interaction of the Martian ionosphere with the solar wind plasma are also a major purpose of the PWS experiment. For these purposes, a wide dynamic range and high sensitivity characteristics of the PWS receiver have been achieved. As the extended function of the PWS plasma sounder instrument, altitude measurement also will be carried out.
\end{abstract}

\section{Introduction}

The Martian ionosphere has a unique structure and dynamics, reflecting the evidence for a direct interaction with the solar wind plasma. Since the first fly-by of Mariner 4 in 1965 , there have been several chances to obtain information about the electromagnetic and plasma environment around Mars. It was found that Mars has an ionosphere with a maximum density of $105 / \mathrm{cc}$ at about $150 \mathrm{~km}$ altitude and a possible weak intrinsic magnetic field of less than 1/1000 of that of Earth (Slavin et al., 1991). Even though there remains the possibility of an extremely weak magnetic field, Mars has no remarkable intrinsic magnetic field to act as a magnetic shielding effect. So, the solar wind plasma can penetrate into the low altitude region of the ionosphere. This feature is one of the most significant characteristics of the Martian upper atmosphere, which is different from that of Earth. As has been confirmed by using Pioneer Venus Orbiter (PVO) data, Venus has an ionosphere associated with a clearly structured ionopause which separates the ionosphere region from the solar wind plasma; the ionopause is understood to be a manifestation of the interaction of the solar wind with the Venusian plasma atmosphere without intrinsic magnetic field (Zhang et al., 1991). Considering the comparison with

Copy right (C) The Society of Geomagnetism and Earth, Planetary and Space Sciences (SGEPSS); The Seismological Society of Japan; The Volcanological Society of Japan; The Geodetic Society of Japan; The Japanese Society for Planetary Sciences. the Venusian ionosphere, the studies of the Martian ionosphere interaction with the solar wind are also significant.

The plasma density profile of the Martian ionosphere has been mainly measured by using an occultation technique. The Viking landers tried to obtain direct measurements of the plasma density profile of the ionosphere by installing a retarding potential probe (Johnson and Hanson, 1991). However, the previous observations of the Martian ionosphere showed no clear evidence of the ionopause structure (Breus et al., 1991); it has been thought to be due to a limitation of the data resolution and the reliability of the observation techniques. Thus, it is desirable to obtain more accurate observation data covering a wide region of the Martian ionosphere to understand the structure and dynamics of the Martian upper atmosphere and to identify the existence of the Martian ionopause. Furthermore, accurate data of the ionosphere structure is thought to be the clue to answering one of the major questions concerning the planet Mars; namely, whether it has an intrinsic magnetic field or not. For this purpose, the observation of electron density profiles from the topside ionosphere of Mars is planned by using the radio wave sounder onboard the Planet-B spacecraft.

Throughout the physical processes associated with the solar wind-ionosphere interaction, strong wave-particle interactions are predicted in the regions of the bow shock, the ionopause, and the ionosphere. Plasma waves are also 
regarded as being the origin of the effective collision of media which produces an effective viscosity and electric resistance as well. Generation of the plasma waves is directly related with the formation of the bow-shock, boundary between the solar wind and the Martian ionosphere, and energy transport through the ionosphere. This fact has been partially verified by the Phobos- 2 orbiter observations within the confines of the limited sensitivity and frequency coverage of the instrumentation (Trotigonen et al., 1991). It is also desired to observe the plasma waves through wider coverage of the Martian upper atmosphere in a wider frequency range and higher sensitivity comparied with the Phobos-2 observations. In the Planet-B orbiter, therefore, the Plasma Waves and Sounder (PWS) instruments are installed with two sets of $52 \mathrm{~m}$ tip-to-tip dipole antennas to carry out extended studies of the structure and dynamics of the Martian ionosphere.

The core part of the PWS system onboard the Planet-B spacecraft, consisting of an ionosphere topside sounder and high frequency plasma wave detectors, is basically the same as the PWS system onboard the Akebono (EXOS-D) (Oya et al., 1990). When we trace back the history of the $\mathrm{RF}$ radio wave sounder for the topside ionosphere, we see that the work had been initiated from the stage of a rocket experiment of a fixed frequency sounding of the ionosphere; and the technique was further developed applying the swept frequency method for a topside sounder satellite (Russell and Zimmer, 1969). The instruments were installed onboard Alouettes I and II; and the experiments were further extended, being installed on the ISIS I and II topside sounder, (Florida, 1969; Franklin and Maclean, 1969; Mar and Garrett, 1969). The first observation across the plasmapause was made by the Jikiken (EXOS-B) satellite (Oya et al., 1981; Oya and Ono, 1987). The first digital system of the plasma sounder was developed in the topside sounder experiment of the Ohzora (EXOS-C) satellite (Oya et al., 1985; Obara and Oya, 1985).

A plasma waves and sounder system consists of two major parts; the first is for a RF sounding of the topside ionosphere of Mars and the second is for high frequency plasma waves in the range from $20 \mathrm{kHz}$ to $5 \mathrm{MHz}$. The observation of the natural plasma waves in the frequency region below $20 \mathrm{kHz}$ is carried out by using a VLF receiver (LFA subsystem). In this paper, the observation method and design of the topside sounding, the instrumentation, and results of function tests are introduced for the PWS system.

\section{Method of Topside Sounding and Instrumenta- tion}

\subsection{Method of topside sounding}

In the present sounder system, RF pulses with a pulse width of $100 \mu \mathrm{sec}$ are fed to a transmission antenna with a tip-to-tip length of $52 \mathrm{~m}$. The RF signal is radiated from the antenna, forming a large amplitude wave packet of the electromagnetic waves in the plasma. As schematically shown in Fig. 1, the plasma waves radiated from the spacecraft propagate downward to the topside region of the planetary ionosphere and make a reflection at a point where the propagating RF frequency matches the cutoff frequencies of the plasma waves; namely $f_{X C}, f_{O C}$ and $f_{Z C}$ defined as:

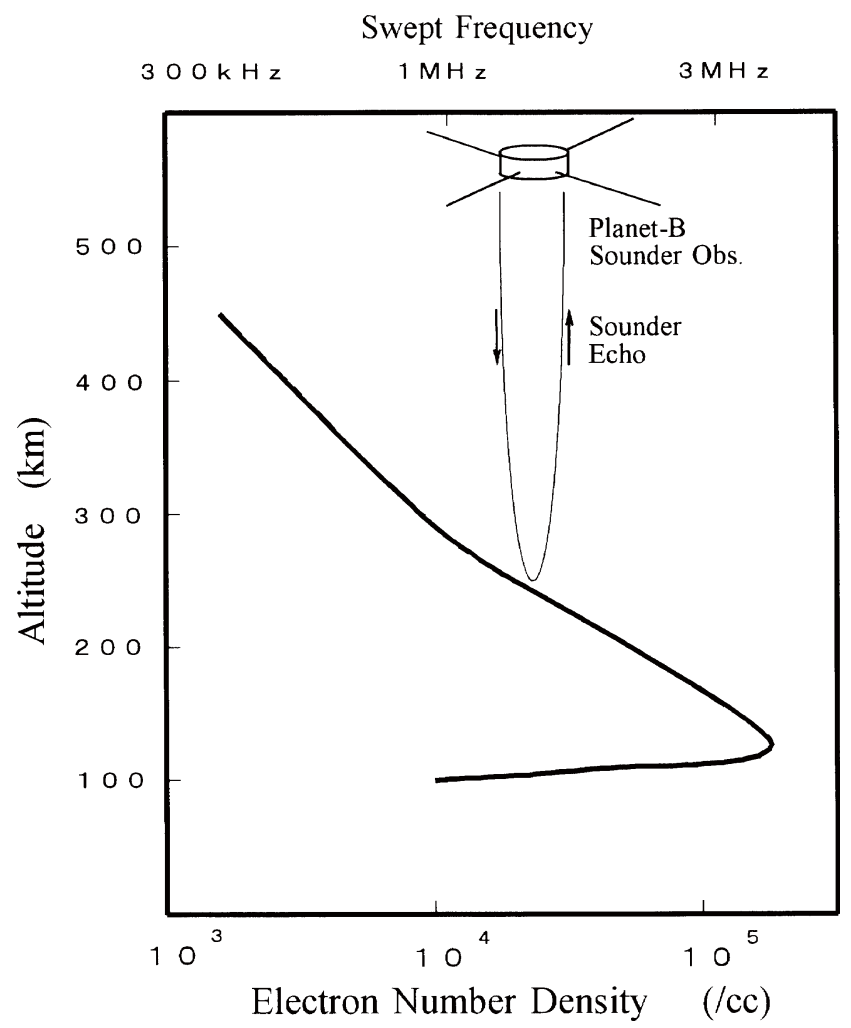

Fig. 1. The concept of topside sounding of the Martian ionosphere by using the PWS plasma sounder experiment system.

$$
\begin{aligned}
& f_{X C}=\frac{\sqrt{4 f_{P}^{2}+f_{H}^{2}}+f_{H}}{2}, \quad f_{O C}=f_{P}, \quad \text { and } \\
& f_{Z C}=\frac{\sqrt{4 f_{P}^{2}+f_{H}^{2}}-f_{H}}{2}
\end{aligned}
$$

for the plasma frequency $f_{P}$ and the electron cyclotron frequency $f_{H}$. Because the intensity of the Martian intrinsic magnetic field is negligibly small, the dispersion relation of the plasma waves is degenerated to that of non magnetized plasma. Therefore, the propagation characteristics of the pulsed plasma waves can be explained by the dispersion relation of $\mathrm{O}$-mode plasma waves with $f_{H} \approx 0$, and the reflection condition can be simplified as taking place $f=f_{O C}$ at at the topside ionosphere. The echo signal returns to the spacecraft with a delay time $\tau(f)$ expressed by;

$$
\tau(f)=2 \int_{h s}^{h r} \frac{1}{V g(f)} d z,
$$

where $h_{r}, h_{s}$ and $\operatorname{Vg}(f)$ are the satellite height, height of the topside reflection point, and the group velocity of the propagating plasma waves with the RF frequency $f$, respectively (Jackson, 1969). The electron density profile along the propagation path of the plasma waves can be obtained by analyzing the relationship between the RF frequency (swept from $50 \mathrm{kHz}$ to $7 \mathrm{MHz}$ ) versus the delay time of the echoes. 


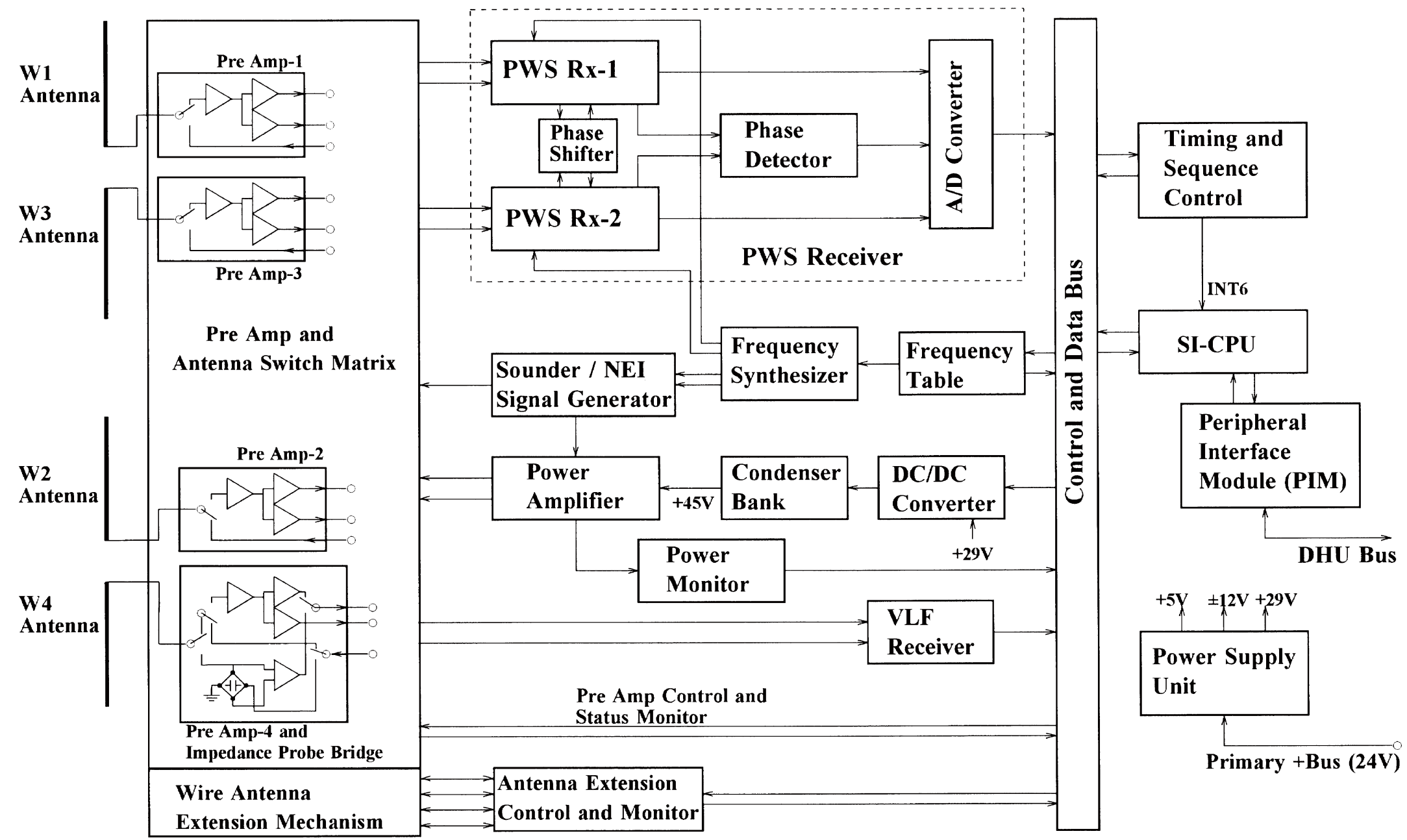

Fig. 2. A block diagram of the PWS instrument. The PWS system consists of two sets of long deployed dipole antennas, four pre-amplifiers, a sounder transmitter, a sounder receiver, a frequency synthesizer, a condenser bank, a power supply unit, and a SI-CPU unit. The main block of the PWS system is assembled together with the plasma wave experiment in the low frequency range (LFA) which is designed separately. 
When the RF frequency is higher than the maximum cutoff frequency of the ionosphere, the RF pulse can penetrate through the ionosphere and it becomes possible to obtain echoes from the Martian surface. In addition to the observation frequency in a range from $50 \mathrm{kHz}$ to $7 \mathrm{MHz}$ for the topside sounding, a frequency range from 8 to $10 \mathrm{MHz}$ is selected for the altimeter observations (ALT mode) for the detection of surface echoes. The method and instrumentation of the altimeter is described in detail in another paper in this issue (Oya and Ono, 1998).

\subsection{Instrumentation}

The instrumental parameters of the whole PWS system are summarized in Table 1. The functions of the passive mode PWS observations are; (1) Observation of dynamic spectra of the plasma waves by using the cross-dipole antennas (DS mode). (2) Observation of the polarization of plasma waves detecting the spectra of right- and left-handed polarized waves at the same time (PL mode). (3) Measurement of the antenna impedance immersed in the Martian ionosphere or solar wind plasma by using the impedance probe technique (NEI mode). The time resolution of passive mode observations such as DS mode, PL mode and NEI mode are 2 seconds to cover the observation frequency swept from $20 \mathrm{kHz}$ to $5 \mathrm{MHz}$. On the other hand, it takes 32 seconds for a complete sweep of the frequency range from $50 \mathrm{kHz}$ to $7 \mathrm{MHz}$ in RF sounder observations.

The block diagram of the PWS system is shown in Fig. 2; the instrumentation consists of wire antennas, pre-amplifiers, a frequency synthesizer, two-stage super heterodyne receivers, a power amplifier subsystem which includes a con- denser bank, and a CPU for controlling the operation of the instruments and onboard data processing.

\section{(1) Antennas}

The PWS system is equipped with two sets of long deployable dipole antennas with a length of $52 \mathrm{~m}$ tip-to-tip. The antenna elements are $\mathrm{BeCu}$ wires with a length of $25 \mathrm{~m}$ and a diameter of $0.38 \mathrm{~mm}$. They are designated $\mathrm{W} 1, \mathrm{~W} 2$, $\mathrm{W} 3$ and $\mathrm{W} 4$ in a clockwise rotation around the Planet-B. The wire antenna modules are mounted on the base-plate of the Planet-B spacecraft separated by the distance of $2 \mathrm{~m}$. Therefore, the total tip-to-tip length of the dipole antenna system is $52 \mathrm{~m}$. For the RF sounder operation, large amplitude RF pulses are normally transmitted from wire antennas W2 and W4. The echo signals are picked up by using the other set of dipole antennas W1 and W3 for the sounder operation.

(2) Power amplifier

For the RF pulse transmission of sounder observations, the RF signal is generated by using a frequency synthesizer. At the power amplifier, pulses from the RF pulse generator are amplified to a final output level of about $720 \mathrm{~V}$ peak-topeak at the antenna feeding point where a shunt load resistor of $100 \Omega$ is set; the output power of the RF pulse is then 650 Watts. The source power of the power amplifier is supplied with a voltage of $45 \mathrm{~V}$ which is fed from a condenser bank with a total capacitance of $4000 \mu \mathrm{F}$. The condenser bank consists of four capacitor blocks with 10 tantalum electrolytic capacitors of $100 \mu \mathrm{F}$, in each block, to watch the reliable operation of the large number of capacitors.

(3) Pre-amplifiers

The pre-amplifier consists of a FET at the front to attain

Table 1. Observation modes and major instrumental parameters of PWS system.

\begin{tabular}{|c|c|c|}
\hline \multirow[t]{5}{*}{ Observation mode and data } & DS mode & Spectral intensities for $X$ and $Y$ crossed dipole antennas \\
\hline & PL mode & $R$ and $L-h a n d e d$ polarized intensities \\
\hline & NEI mode & Equivalent capacitance of $\mathrm{W}-4$ antenna and plasma wave spectrum for $Y$-antenna \\
\hline & SPW mode & $\begin{array}{l}\text { Pulse transmission and detection of the pulse intensity of an echo from the } \\
\text { topside ionosphere }\end{array}$ \\
\hline & ALT mode & $\begin{array}{l}\text { Pulse transmission of both sideband signals and detection of pulse intensity and } \\
\text { phase lag of surface echoes }\end{array}$ \\
\hline \multirow[t]{3}{*}{ Antenna } & \multicolumn{2}{|c|}{2 sets of $52 \mathrm{~m}$ tip-to-tip dipole antennas } \\
\hline & $X$-antenna: & W-2 and W-4 pair (transmission antenna for the normal case) \\
\hline & $Y$-antenna: & W-1 and W-3 pair (receiving antenna for the normal case) \\
\hline \multirow[t]{5}{*}{ Power amp and RF pulse generator } & Frequency range & $50 \mathrm{kHz}$ to $10 \mathrm{MHz}$ \\
\hline & Output power & 400 Watts for $200 \Omega$ load resistance \\
\hline & Pulse width & $100 \mu$ sec for SPW mode \\
\hline & & $300 \mu$ sec for ALT mode \\
\hline & Pulse repetition & $125 \mathrm{msec}$ \\
\hline
\end{tabular}

Pre-amp.

Receiver
$100 \mathrm{M} \Omega$ input impedance

$\begin{array}{ll}\text { Dynamic range } & -133 \mathrm{dBV} \sim-13 \mathrm{dBV} \text { (gain control; H, M, L modes) } \\ \text { Frequency range } & 20 \mathrm{kHz}-10 \mathrm{MHz} \text { (software program control) } \\ \text { Sweep time } & 2 \mathrm{sec} \text { for passive mode observation (DS, PL, NEI modes) } \\ & 32 \mathrm{sec} \text { for swept frequency sounder mode (SPW mode) } \\ & \pm 2.4 \mathrm{kHz}(-3 \mathrm{~dB}) \text { for passive mode (DS, PL, NEI modes) } \\ \text { Band width } & \pm 20 \mathrm{kHz}(-3 \mathrm{~dB}) \text { for active mode (SPW, ALT modes) } \\ & 7.81 \mathrm{msec} \text { for DS, PL, and NEI modes data } \\ \text { Data sampling } & 25 \mu \mathrm{sec} \text { for SPW and ALT modes data }\end{array}$


a high input impedance of the pre-amplifier, followed by a buffer amplifier which divides the signals into the high and low frequency parts. The signal in the higher frequency part, covering the frequency range above $20 \mathrm{kHz}$, is fed to the main amplifier of the PWS receiver.

\section{(4) Receivers}

Two receiver modules are installed in the PWS system, as shown in Fig. 2. For passive mode observation, as well as ALT-mode observation, both PWS Rx-1 and PWS Rx-2 receivers are operated at the same time. On the other hand, for the plasma sounder operation, the PWS Rx-1 receiver is operated for detecting topside echoes with a high time resolution. The main receivers, PWS Rx-1 and PWS Rx-2, consist of a two-stage super heterodyne system where the center frequency of the first stage amplifier is set at $25 \mathrm{MHz}$, while the center frequency of the second IF stage (intermediate frequency stage) is set at $455 \mathrm{kHz}$. To obtain a high range resolution for the sounder measurements, the shape of the receiver output pulse is required to be sharp; the band width of the sounder receiver is therefore selected to be a wide width of $\pm 32 \mathrm{kHz}$ for the first IF stage and $\pm 20 \mathrm{kHz}$ for the second IF stage. The echo signals converted to the second IF frequency $(455 \mathrm{kHz})$ is fed to the AC logamplifier which converts an analog AC signal with a wide dynamic range of more than $70 \mathrm{~dB}$ to a logarithm-compressed signal with an amplitude range of about $50 \mathrm{~dB}$. After logarithm compression, the $455 \mathrm{kHz}$ signal amplitude is measured by using precision detectors and fed to the analogto-digital (AD) converter.

\section{(5) Frequency synthesizer}

Conversions of the frequency of the signals fed from the pre-amplifier are made by applying local frequency signals generated through direct digital synthesizers. To achieve the frequency spectrum analyzer in a range from $20 \mathrm{kHz}$ to 5 $\mathrm{MHz}$ for passive mode observation and from $50 \mathrm{kHz}$ to 7 $\mathrm{MHz}$ for the topside sounder mode operation, the frequency of the local signal for conversion to a first-stage signal is swept from 25.020 to $30.000 \mathrm{MHz}$ with time interval of 2 sec, and from 25.050 to $32.000 \mathrm{MHz}$ with time interval of 32 $\mathrm{sec}$, respectively. The generation of the local frequency is controlled by a CPU where we can select a wide variety of frequency ranges following the frequency table in the synthesizer. The frequency table is also rewritable by using CPU software with $1 \mathrm{kHz}$ resolution. To generate the RF frequencies of the sounder signals, the synthesized signals are mixed with a $25 \mathrm{MHz}$ signal, and converted to a RF frequency swept from $50 \mathrm{kHz}$ to $7 \mathrm{MHz}$. This conversion process is also used to obtain the swept frequency signal for the NEI observation.

\section{(6) Onboard data processing}

To obtain a high range resolution for the sounder measurement, the sounder receiver has a high speed AD conversion speed of $25 \mu \mathrm{sec}$ which is equivalent to a range resolution of $3.75 \mathrm{~km}$ for the echo observation. The repetition period of the sounder pulse transmission is $125 \mathrm{msec}$. It takes $32 \mathrm{sec}$ for complete coverage of a swept frequency range with 256 steps of frequency variation. During this time, the spacecraft traverses about $150 \mathrm{~km}$ near the periapsis of the orbit; then a range of $150 \mathrm{~km}$ becomes the maximum spatial resolution of the sounder ionogram data. Under the limitation of the handling data rate and telemetry speed, the allocated data rate for the sounder experiment is below 1 kbytes/sec. A CPU program is then constructed to optimize the data to fit the observation conditions and available data rate, mostly by compressing the 8 bit original codes into 4 bit.

\section{Verified Performance of the PWS System 3.1 RF sounder power transmission}

The final stage of the power amplifier of the RF sounder system is given in Fig. 3. The four power transistors are operated in parallel; two pairs of power transistors amplify

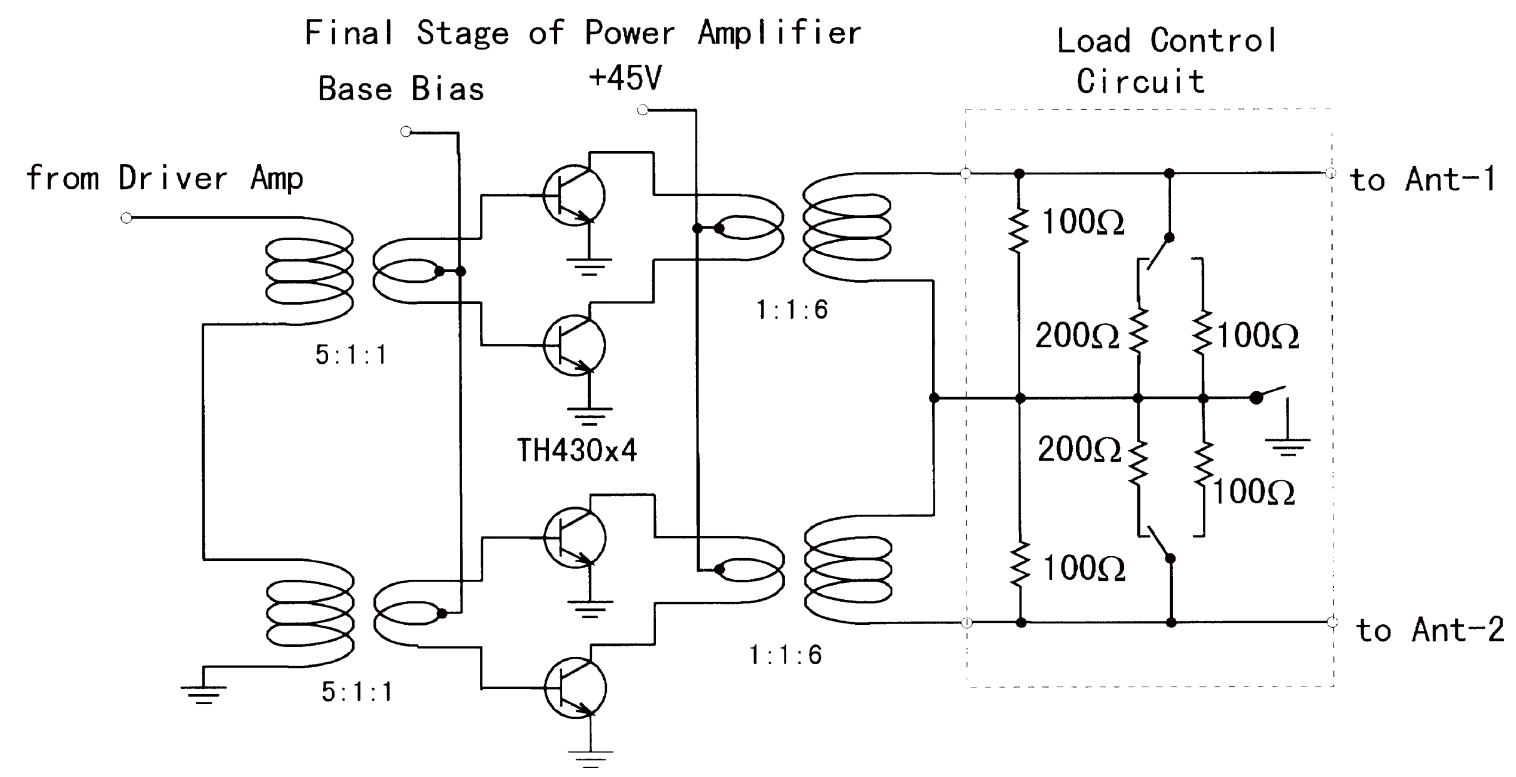

Fig. 3. The circuit diagram of the final output stage of the power amplifier. The output transformer is connected to the load control unit which can select the shunt load values and the ground (spacecraft body) connection condition of the center tap of the transformer to maintain the stability of the high power amplifier. 


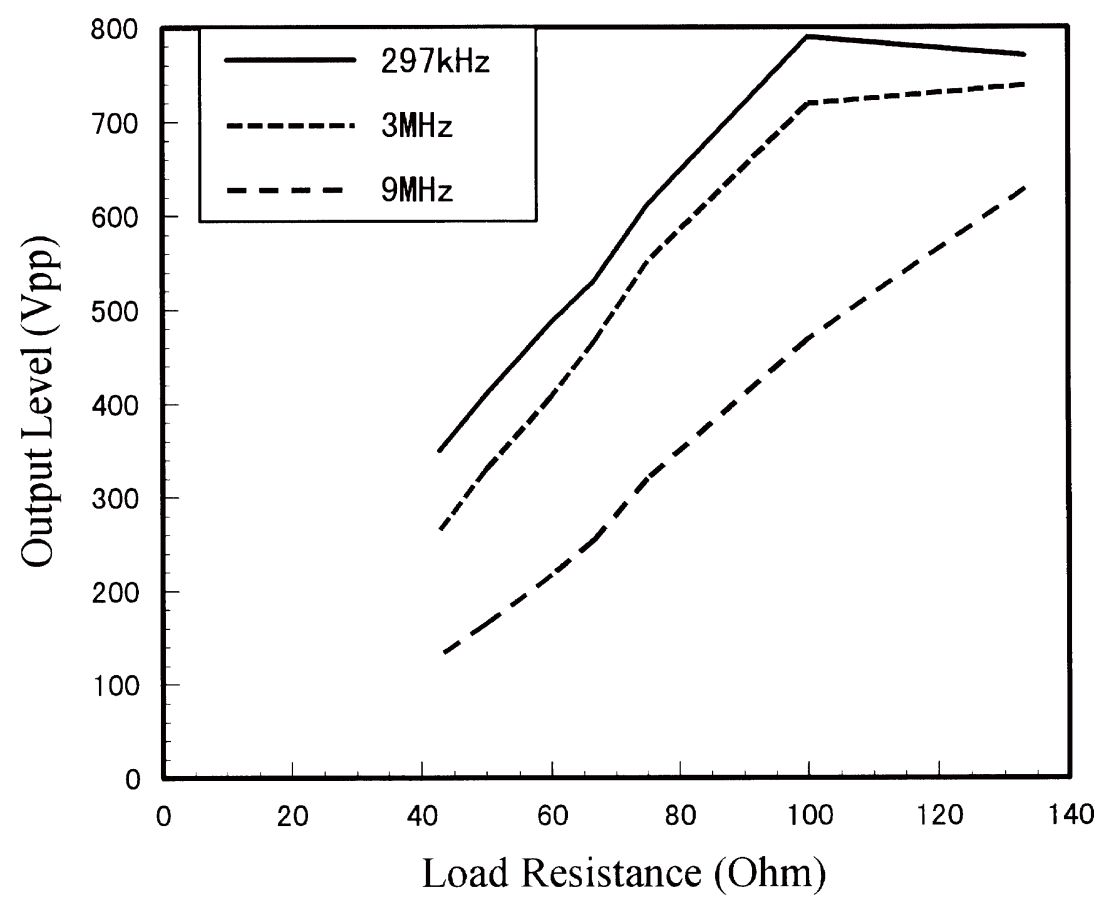

Fig. 4. Output signal level of the power amplifier versus total load resistance. Dependence on the load resistance is given for example frequencies at $297 \mathrm{kHz}, 3 \mathrm{MHz}$, and $9 \mathrm{MHz}$.

the power of RF pulse signals, making a push-pull arrangement. The effective performance of the same-type power amplifiers has already been verified through three past satellites observations by Jikiken (EXOS-B), Ohzora (EXOSC) and Akebono (EXOS-D), respectively, launched in 1978, 1984, and 1989 (Oya et al., 1981, 1985, 1990). In Fig. 4, the output voltages given by the peak-to-peak value versus the load resistance are plotted for the present power amplifier prepared for the present system, taking selected example frequencies at $297 \mathrm{kHz}, 3 \mathrm{MHz}$ and $9 \mathrm{MHz}$. As has been given in Fig. 3, the load resistance is set in parallel with the transmission antenna, for the purpose of obtaining the stabilization of the power amplifier. This method makes it possible to keep the power amplifier stable, when there is a large amount of antenna impedance variation during operating frequencies. Because the antenna length is fixed in space plasma, the real and imaginary parts of antenna impedance show strict frequency dependence. It is, then, impossible to maintain the complete adjustment of the antenna impedance to match with the output impedance of the power amplifier at each operation frequency. As shown in Fig. 4, an output signal amplitude of $720 \mathrm{~V}_{\mathrm{p}-\mathrm{p}}$ is obtained for a load impedance of $100 \Omega$ (650 Watts) for a frequency of $3 \mathrm{MHz}$. When the $52 \mathrm{~m}$ dipole antenna is connected, the dipole impedance becomes $75 \Omega$ at $3 \mathrm{MHz}$ because of matching the antenna length with a $\lambda / 4$ condition with respect to the wavelength $\lambda$. In this case, the total load impedance applied to the power amplifier becomes $48 \Omega$. As we can see in Fig. 4 again, the output amplitude at the antenna becomes $340 \mathrm{~V}_{\mathrm{p} \text {-p }}$ generating $196 \mathrm{Watts}$ for radiation power.

\subsection{Reception of the transmitted pulse}

The received pulse power $P_{R}$ can be roughly estimated by using the following radar equation (Franklin and Maclean, 1969),

$$
P_{R}=\frac{G^{2}}{L} P_{T}\left\{\frac{\lambda}{4 \Pi(2 R)}\right\}^{2}
$$

where $L, G, \lambda, R$ and $P_{T}$ are a power loss factor, the antenna gain, the sounding wavelength, distance between the transmitting antenna and reflection point (the propagation range of echoes), and the power fed to the antenna element, respectively. For $3 \mathrm{MHz}$ waves, a dipole antenna with a 52 $\mathrm{m}$ tip-to-tip length has a gain of $2.2 \mathrm{~dB}$; the loss rate can be set within $-10 \mathrm{~dB}$, then the possible receiving power can be estimated as $2.1 \times 10^{-10}$ Watt inducing antenna voltage of $78 \mathrm{dBV}$ at a matched dipole antenna of $3 \mathrm{MHz}$ for $2000 \mathrm{~km}$ range propagation. This echo signal is strong enough to be detected by the PWS receiver whose response is shown in Fig. 5 where the receiver output levels are indicated versus the input signal level diagram for three operational gains at high (H), medium (M) and low (L) levels. From Fig. 5, it becomes clear that the signal can be detected by the sounder receiver with medium gain. As has been calculated by Franklin and Maclean (1969), and discussed by Obara and Oya (1985), the mismatch loss due to a variation of an antenna impedance in space is unavoidable. When the frequency changes to $300 \mathrm{kHz}$, which is a very possible frequency range for sounding the Martian ionopause, the antenna impedance in the vacuum takes a resistance of 0.6 $\Omega$ and reactance of $-7.5 \mathrm{k} \Omega$ for example. In this case, the amplitude of the radiation component of the electric field decreases about $82 \mathrm{~dB}$ from the matching case due to the antenna impedance mismatch. Even though, in this case, the echo signal strength with an apparent range of $300 \mathrm{~km}$ is estimated to be $-114 \mathrm{dBV}$, which is still in the dynamic range of the PWS receiver. 


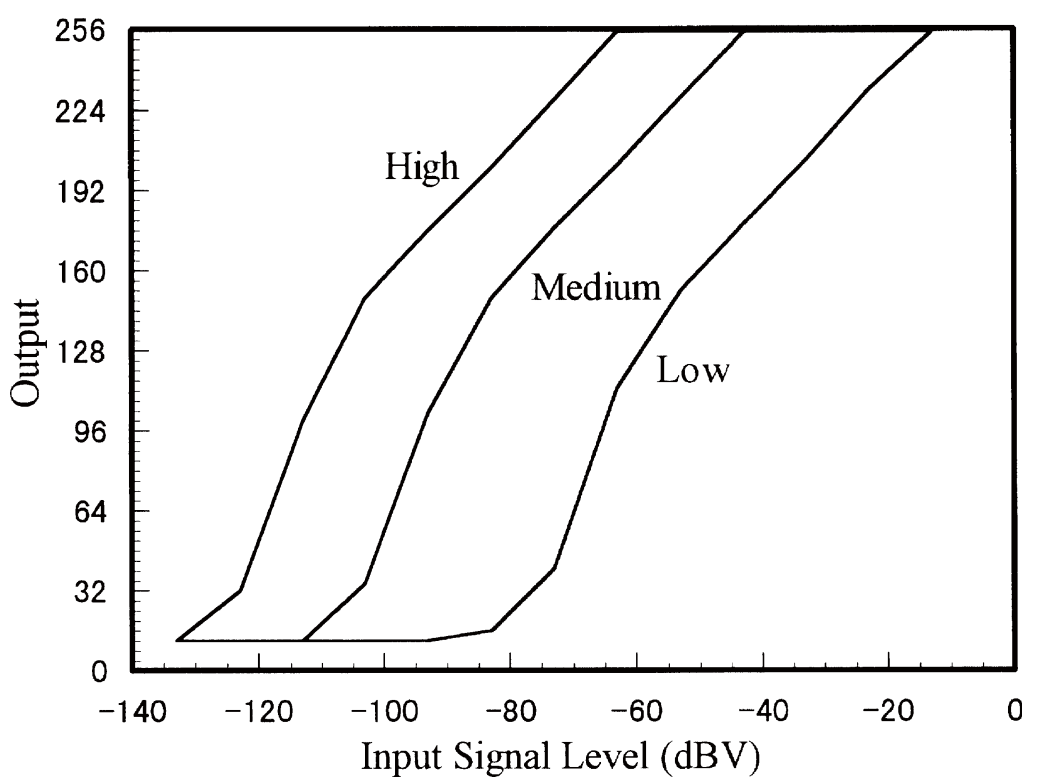

Fig. 5. The output level of the PWS receiver given by PCM value versus input signal level at the pre-amplifier for High gain (H), Medium gain (M) and Low gain (L) mode operations for passive mode operations. The input level is given in $\mathrm{dBV}$ (i.e. $0 \mathrm{dBV}=1 \mathrm{Vrms}$ ).

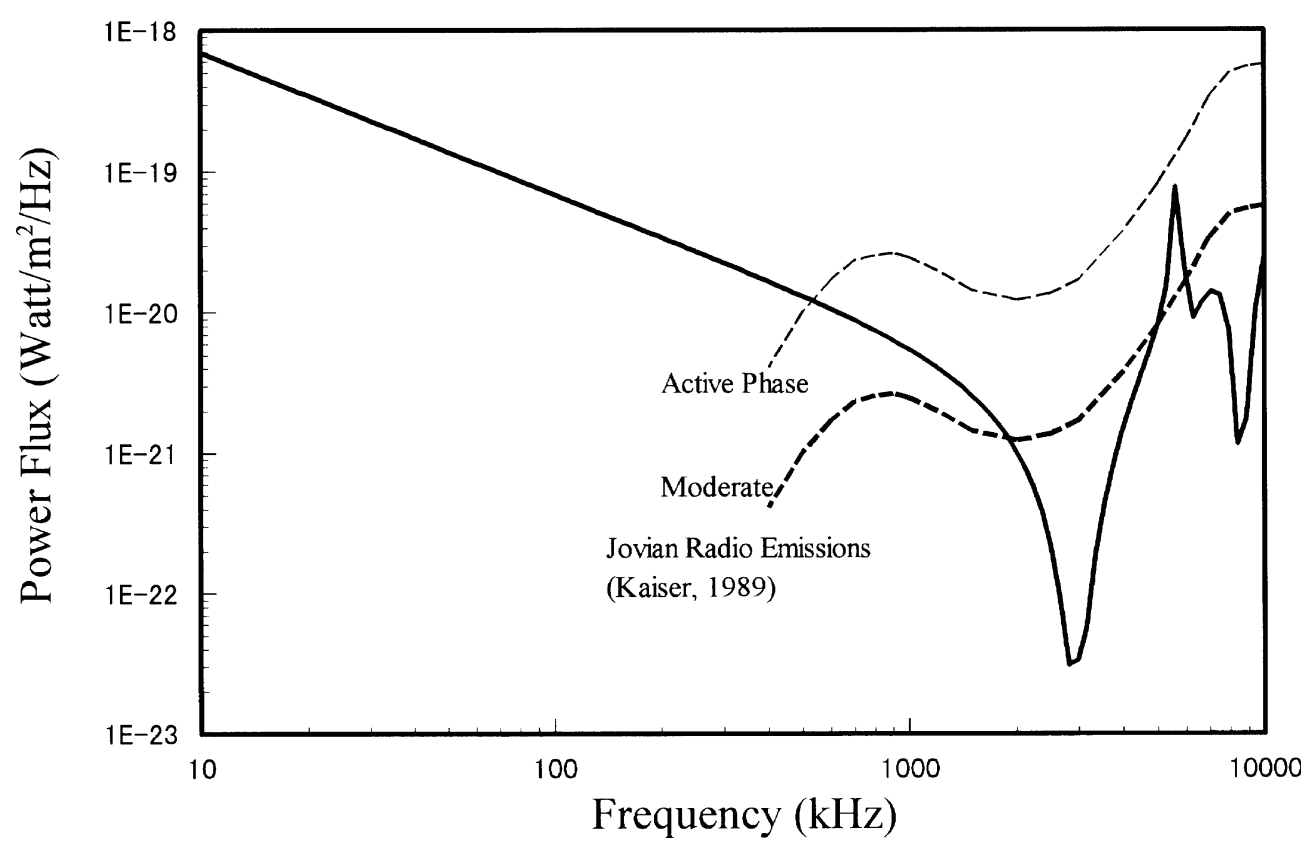

Fig. 6. The threshold of the PWS passive receiver given by the equivalent power flux of radio waves in units of Watt $/ \mathrm{m}^{2} / \mathrm{Hz}$. The receiver threshold is compared with the moderate state power flux of the Jovian hectometric and decametric radiations proposed by Kaiser (1989) for an observation distance of 3.8 AU from Jupiter. For the case of the active state of Jovian radio emission, the threshold is further compared with Jovian radio emission of the active phase enhanced by $10 \mathrm{~dB}$ from the moderate emission level (thin dotted curve).

\subsection{Plasma wave receiver}

The Phobos-2 orbiter reported the generation of strong plasma waves associated with bow shock traverses in the orbit. To obtain an accurate spectra of the plasma waves in these turbulent regions, the PWS receiver has a wide dynamic range response. When the receiver is operated in the low gain mode, a spurious free receiver response can be obtained up to a pre-amplifier input level of $0.21 \mathrm{~V}_{\mathrm{p}-\mathrm{p}}$.

On the other hand, the PWS receiver has high sensitivity characteristics for the observation of a planetary radio waves. As given in Fig. 6, an equivalent power flux of the system noise level for a high gain operation is obtained considering that FET at the pre-amplifier signal is an equivalent current noise source with the noise level depending only on the impedance at the input point of the preamplifier under the condition that the antenna is connected to the pre-amplifier. Figure 6 shows that there is a window between 2 and $5 \mathrm{MHz}$ where the threshold is around $10^{-21}$ Watt $/ \mathrm{m}^{2} / \mathrm{Hz}$ which is well below the estimated intensity of the moderate state of the Jovian hectometric and decametric radio emissions for the case of normal spectra proposed by Kaiser (1989) and Gurnett (1995). When these radio emis- 


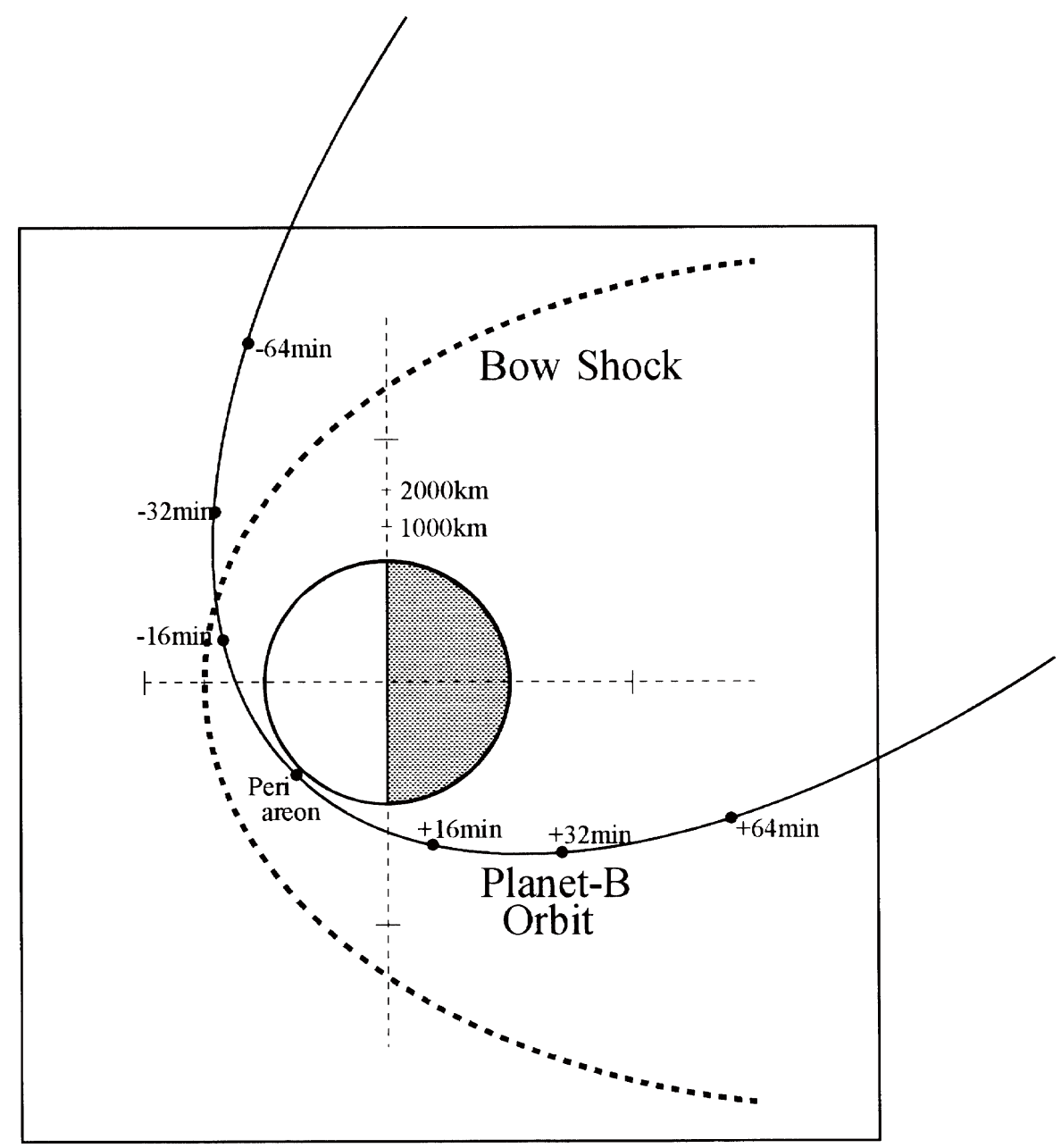

Fig. 7. Depiction of the Planet-B orbit for an example period near the peri-areon with the possible position of the Martian bow shock.

sions become active, a fairly wide frequency range of radio emissions may be observed by using the PWS receiver, as indicated by the thin dashed line in Fig. 6, for enhanced radio emissions $10 \mathrm{~dB}$ greater than the moderate levels of Jovian radio emissions. Type III solar radio bursts are also possible to be observed within the frequency range from $100 \mathrm{kHz}$ to $10 \mathrm{MHz}$, as have been frequently observed by previous PWS system satellite observations (Oya et al., 1990). Because there is no intense auroral activity in the Martian ionosphere and magnetosphere, radio wave emissions around Mars are relatively calmer than around the Earth. So planetary radio wave observation using the PWS system from the Mars orbit is expected to be able to be carried out with a high signal-tonoise ratio.

\section{Observation Plan}

After the orbital insertion of the Planet-B spacecraft into the Martian orbit, a wire antenna will be deployed to form two orthogonally arranged sets of dipole antenna systems; preparation for the instrumental operation will be made by computer (CPU) aided processes. The initial orbital elements are planned to have peri-areon altitude, apo-areon distance and the orbital period of $300 \mathrm{~km}, 15$ Martian radii and 38 hours, respectively. A rough concept of the orbit is given in Fig. 7. The peri-areon altitude will be decreased down to 150 $\mathrm{km}$ if it becomes possible. Due to the highly eccentric nature of the orbit, ionosphere sounding will be possible around the time period when the Planet-B approaches the peri-areon. As shown in Fig. 7, Planet-B passes through the bow shock (position is given following Slavin et al., 1991) and the ionosphere region in less than 2 hours. Considering this point, the PWS observation plan will be set, depending upon the orbital altitude of the spacecraft, as follows: (1) In the low altitude region below $300 \mathrm{~km}$, altimeter (ALT mode) observations will be carried out to measure the surface condition of Mars. (2) In the altitude region from 300 to $3000 \mathrm{~km}$, topside soundings (SPW mode) will be made to obtain an electron density profile of the Martian topside ionosphere. (3) Around the bow shock region, the passive mode of observations for plasma and plasma wave measurements (DS mode, PL mode and NEI mode) will be made, focussing on the observation of the plasma disturbances and wave-particle interactions associated with the contact of solar wind plasma with the ionosphere plasma. (4) Far outside the region of the Martian ionosphere, study of the planetary radio waves and plasma waves in the solar wind will be carried out by using the passive observation mode of the PWS system (DS mode and PL mode).

\section{Conclusion}

The Planet-B mission for exploring the Martian upper atmosphere was started in 1992. The mission objective is to 
understand the structure and dynamics of the Martian upper atmosphere under the condition of the direct interaction with the solar wind. The PWS instrument is designed to observe the structure of the ionosphere by using the topside sounder technique together with the features of plasma waves associated with wave particle interactions which become the origin of the microprocesses controling the direct interaction processes of the ionospheric plasma with the solar wind plasma. Including the two sets of long deployed dipole antennas with a $52 \mathrm{~m}$ tip-to-tip length, a PWS instrument has been established with total weight of $7.81 \mathrm{~kg}$. The basic characteristics of the PWS power amplifier and receiver have been verified after testing the functions, the space plasma simulation chamber test, and environmental tests for launch and orbital conditions; it is concluded that the sounder echoes from the topside ionosphere below a height of 3000 $\mathrm{km}$ can be detectable. The wide dynamic range of the sounder receiver covers not only the detection of the echo signal level but also the detection of the natural plasma waves associated with the direct interaction of the solar wind with Martian ionosphere. The planetary radio emissions from Jupiter and other planets can also be detected by using a low-noise PWS receiver. The observation of the Martian upper atmosphere by using PWS system will give us a new understanding of the upper atmosphere of Mars which is characterized as a planet without a remarkable magnetic field or a very weak intrinsic-magnetic field.

Acknowledgments. The Planet-B mission has been organized by the Institute of Space and Astronautical Science (ISAS) through the efforts of the working group studies on the mission to the terrestrial planets. The authors thank all members of the working group who established the selfless decision to go to Mars. The authors are also grateful to Profs. K. Tsuruda, I. Nakatani and T. Yamamoto for their efforts in carrying out the management of the mission. The manufacturing of the PWS instrument was carried out by Meisei Electric Co. The authors express their thanks to Mr. I. Tanaka and all the engineers of the Space Development Branch of Meisei. The effective arrangements for carrying out the systems function test of Planet-B was conducted by Mr. Adachi of the NEC company; the present authors express their thanks for his efforts.

\section{References}

Breus, T. K., A. M. Krymskii, R. Lundin, E. M. Dubinin, J. G. Luhmann, Ye. G. Yereshenko, S. V. Barabash, V. Ya. Mitnitskii, N. F. Pissarenko, and V. A. Styashkin, The solar wind interaction with Mars: Consideration of PHOBOS 2 mission observation of an ion composition bound- ary on the dayside, J. Geophys. Res., 96, A7, 11165-11174, 1991.

Florida, C. D., The development of a series of ionospheric satellite, Proc. IEEE, 57, 867-875, 1969.

Franklin, C. A. and M. A. Maclean, The design of swept-frequency topside sounders, Proc. IEEE, 57, 897-944, 1969.

Gurnett, D. A., Solar system plasma waves, Radio Sci. Bulletin, 274, 413, 1995.

Jackson, J. E., The reduction of topside ionograms to electron density profiles, Proc. IEEE, 57, 960-976, 1969.

Johnson, F. S. and W. B. Hanson, Viking 1 electron observation at Mars, J. Geophys. Res., 96, A7, 11097-11108, 1991.

Kaiser, M. L., Observation of non-thermal radiation from planets, in Plasma Waves and Instabilities at Comets and in Magnetosphere, edited by B. T. Tsurutani and H. Oya, AGU Geophysical Monograph 53, 221237, 1989.

Mar, J. and T. Garrett, Mechanical design and dynamics of the Alouette spacecraft, Proc. IEEE, 57, 882-896, 1969.

Obara, T. and H. Oya, Plasma condition in the polar ionosphere in SPW experiments of PPS system on board the Ohzora (EXOS-C) satellite, $J$. Geomag. Geoelectr., 37, 285-307, 1985.

Oya, H. and T. Ono, Stimulation of plasma waves in the magnetosphere using JIKIKEN (EXOS-B) satellite, Part II: Plasma density across the plasmapause, J. Geomag. Geoelectr., 39, 591-607, 1987.

Oya, H. and T. Ono, A new altimeter for mars land shape observation utilizing the ionospheric sounder system onboard the Planet-B spacecraft, Earth Planets Space, 50, this issue, 229-234, 1998.

Oya, H., T. Ono, and T. Kamada, Stimulation of plasma waves in the magnetosphere using satellite JIKIKEN (EXOS-B), Part-I: Observation of plasma resonances, J. Geomag. Geoelectr., 33, 3-25, 1981.

Oya, H., A. Morioka, and T. Obara, Leaked AKR and terrestrial hectometric radiations discovered by the plasma wave and planetary plasma sounder experiments on board the Ohzora (EXOS-C) satellite-Instrumentation and observation results of plasma wave phenomena, J. Geomag. Geoelectr., 37, 237-262, 1985.

Oya, H., A. Morioka, K. Kobayashi, M. Iizima, T. Ono, H. Miyaoka, T. Okada, and T. Obara, Plasma wave observations and sounder experiments (PWS) on board Akebono (EXOS-D) satellite, J. Geomag. Geoelectr., 42, 411-442, 1990.

Russell, S., II and F. C. Zimmer, Development of the fixed frequency topside-sounder satellite, Proc. IEEE, 57, 876-881, 1969.

Slavin, J. A., K. Schwingenschuh, W. Riedler, and Ye. Yeroshenko, The solar wind interaction with Mars: Mariner 4, Mars 2, Mars 3, Mars 5, and Phobos 2 observations of bow shock position and shape, J. Geophys. Res., 96, A7, 11235-11241, 1991.

Trotigonen, J. G., R. Grard, and S. Savin, Plasma wave system measurements of the Martian bow shock from the Phobos 2 spacecraft, $J$. Geophys. Res., 96, A7, 11253-11264, 1991.

Zhang, T. L., J. G. Luhmann, and C. T. Russell, The magnetic barrier at Venus, J. Geophys. Res., 96, 11145-11153, 1991.

T. Ono (e-mail: ono@ stpp3.geophys.tohoku.ac.jp), H. Oya, A. Morioka, A. Kumamoto, K. Kobayashi, T. Obara, and T. Nakagawa 